\title{
Diversity in rhynchocephalian Clevosaurus skulls based on CT reconstruction of two Late Triassic species from Great Britain
}

Sofia A.V. Chambi-Trowell, David I. Whiteside, and Michael J. Benton

Acta Palaeontologica Polonica 64 (1), 2019: 41-64 doi:https://doi.org/10.4202/app.00569.2018

Clevosaurus was an early sphenodontian, known from many specimens and species from the Late Triassic and Early Jurassic. Here we describe and compare the cranial morphology of Clevosaurus hudsoni, the type species, and Clevosaurus cambrica, both from the UK, based on digital reconstructions from segmented CT scan data of two skulls. $C$. hudsoni has been described in some detail, but the CT data reveal new information on cranial anatomy, and C. cambrica is a newly described species for which the CT scans enable us to identify many previously undescribed bones, re-identify bones whose identity had previously been uncertain, and refine certain cranial elements. The near complete preservation of the left side of the skull of $C$. cambrica, and the preservation of much of the left side of the skull of $C$. hudsoni, provided us with the opportunity to fully reconstruct both species' skulls in three dimensions. For the first time, coronoid bones are identified in Clevosaurus species from the British Isles. These two species show the diversity of morphology in the genus Clevosaurus. We provide evidence for two morphotypes of $C$. hudsoni based on study of the dentary dentition of the syntypes and other attributed specimens.

Key words: Rhynchocephalia, Lepidosauria, Clevosaurus, anatomical description, 3D skull reconstruction, CT scanning, Triassic, UK.

Sofia Chambi-Trowell [sc14927@my.bristol.ac.uk], David I. Whiteside [David.Whiteside@bristol.ac.uk], and Michael J. Benton [mike.benton@bristol.ac.uk], School of Earth Sciences, University of Bristol, Bristol, BS8 1TF, UK.

This is an open-access article distributed under the terms of the Creative Commons Attribution License (for details please see creativecommons.org), which permits unrestricted use, distribution, and reproduction in any medium, provided the original author and source are credited. 
Porif Full text $(2,046.5 \mathrm{kB})$ ।

POFF Supplementary file $(1,869.4 \mathrm{kB})$ 